\title{
Adaptive interferometry of protein on a BioCD
}

\author{
Leilei Peng, ${ }^{1,3, \star}$ Manoj M. Varma, ${ }^{1}$ Wonryeon Cho, ${ }^{2}$ Fred E. Regnier, ${ }^{2}$ and David D. Nolte ${ }^{1}$ \\ ${ }^{1}$ Department of Physics, Purdue University, 525 Northwestern Avenue, West Lafayette, Indiana 47907, USA \\ ${ }^{2}$ Department of Chemistry, Purdue University, 560 Oval Drive, West Lafayette, Indiana 47907, USA \\ ${ }^{3}$ Present address is Wellman Center for Photomedicine, Massachusetts General Hospital, \\ Harvard Medical School, Boston, Massachusetts 02114, USA
}

${ }^{*}$ Corresponding author: Ipeng1@partners.org

Received 26 February 2007; accepted 18 April 2007;

posted 11 May 2007 (Doc. ID 80351); published 23 July 2007

\begin{abstract}
Adaptive spinning-disk interferometry is capable of measuring surface profiles of a thin biolayer with subnanometer longitudinal resolution. High-speed phase modulation in the signal beam arises from the moving surface height profile on the spinning disk and is detected as a homodyne signal via dynamic two-wave mixing. A photorefractive quantum-well device performs as an adaptive mixer that compensates disk wobble and vibration while it phase-locks the signal and reference waves in the phase quadrature condition ( $\pi / 2$ relative phase between the signal and local oscillator). We performed biosensing of immobilized monolayers of antibodies on the disk in both transmission and reflection detection modes. Single- and dual-analyte adaptive spinning-disk immunoassays were demonstrated with good specificity and without observable cross-reactivity. Reflection-mode detection enhances the biosensing sensitivity to one-twentieth of a protein monolayer, creates a topographic map of the protein layer, and can differentiate monolayers of different species by their effective optical thicknesses. (C) 2007 Optical Society of America
\end{abstract}

OCIS codes: $170.3890,160.5320,120.3180$.

\section{Introduction}

Optical interference can measure optical path changes with longitudinal resolutions comparable to or better than the most sensitive surface-scanning microscopic techniques, such as atomic force microscope. However, measuring optical path changes requires the interferometer to maintain the quadrature condition where the relative phase between the probe and the local oscillator is equal to $\pi / 2$. In surface-scanning interference microscopy, the path length difference between the signal and reference beams must be stabilized to the subnanometer level in order to detect nanometer-size features (for example protein molecules). We previously demonstrated several free-space interferometric techniques with such capability. One technique is called self-referencing spinning-disk interferometry [1], that sets the quadrature condition using micro-structures on a reflective surface. Another technique uses an adaptive interferometer to detect proteins on a transpar-

0003-6935/07/220001-12\$15.00/0

(C) 2007 Optical Society of America ent surface [2]. In this paper, we further report the use of adaptive free-space interferometry to quantify the optical thickness of nanometer-thin patterns on both transparent and reflective surfaces. The results demonstrate that adaptive interferometry can provide both precise characterization of thin layers and quantified detection of biomolecules.

Adaptive optical interferometry [3-7] uses an adaptive beam mixer, for example photorefractive materials, instead of a beamsplitter to generate interference between a local oscillator and signal beam. By automatically compensating time and spatial perturbations in the input fields, a steady quadrature condition can be maintained in an adaptive interferometer, which enables pure phase-sensitive detection, such as ultrasound detection [8-12]. Subpicometer surface displacement sensitivity have been demonstrated with homodyne detection in photorefractive materials $[9,13]$. Such sensitivity is much smaller than the roughness of an atomically flat surface and is sufficient for biomolecule pattern recognition. If one detects the interference signal on the local oscillator arm instead of the usual signal arm, adaptive interferometric de- 
tection can also avoid intensity noise in the signal beam.

To make stationary features on a sample "visible" to adaptive interferometry, phase changes caused by spatially-distributed surface features must be converted to a temporal frequency higher than the compensation rate of the adaptive mixer. In principle, one could scan the detection beam to achieve such a high frequency signal. However, it is easier and cheaper to move the sample, and the speed and movement range are much larger. Inspired by the compact disk (CD) technology, a disk readout scheme is used in which biomolecule patterns are immobilized on a disk platform. By spinning the disk at high speed, static patterns are converted to high-frequency signals, which are away from $1 / f$ noise and have a higher signalto-noise ratio than for static measurements. This method gives the system the ability to detect multiple analytes by specifically allocating different parts of the disk surface to different analytes as a type of molecular random-access addressing. Another advantage of the spinning-disk scheme is its high readout speed, which can cover the entire disk in tens of minutes, as a CD player does. The same scheme also has been used with self-referencing interference [1]. Because of their similarity to the optical CD, adaptive and self-referencing spinning-disk interferometries emerge as a new group of biosensing techniques referred as the BioCD.

\section{Intensity-Free Homodyne Detection Using Photorefractive Quantum Wells}

Photorefractive quantum well (PROW) [14] devices are used as the mixing element in adaptive spinningdisk interferometry. These devices self-compensate thermal and mechanical disturbances and maintain the quadrature condition with a compensation rate higher than a $\mathrm{kHz}$ [15]. Phase modulation in the probe beam at frequencies higher than the compensation rate of the PRQW device is read out by a photodetector as intensity modulation caused by interference between the transmission (zero-order) of one beam and the first-order diffraction of the other beam. The two-wave mixing signal after a PRQW device is given by

$$
\Delta P_{p, l} \approx 2 \sqrt{P_{p}^{0} P_{l}^{0}} \cos \left[\varphi(t)+\varphi_{P}+\psi(\omega)+\frac{\pi}{2}\right]
$$

neglecting a common overall absorption of the beams in the PRQW, where $P_{l}^{0}$ and $P_{p}{ }^{0}$ are the probe and local oscillator power before the PRQW, $\varphi(t)$ is the phase modulation, and $\varphi_{P}$ is the photorefractive phase shift that represents the phase offset between the intensity pattern and the optical index pattern driven by the space-charge formation $[15,16]$. The excitonic spectral phase $\psi(\omega)$ is defined by

$$
\frac{\mathrm{d} \psi}{\mathrm{d} \omega}=T_{2}
$$

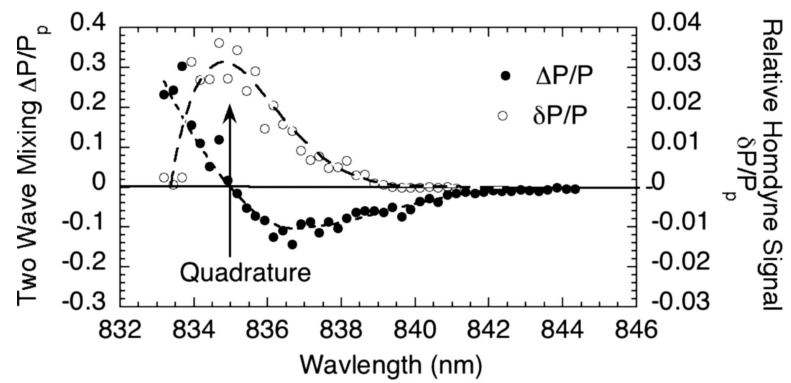

Fig. 1. Two-wave mixing and homodyne signals of a PRQW device (BH51). The homodyne signal is measured with a $5-\mathrm{MHz}$ $\Delta_{\varphi}=\frac{\pi}{6}$ phase modulation. The intensity ratio $\beta$ between the probe and local oscillator beam is 4 . Homodyne power is calibrated to be $\frac{\delta P_{p}}{P_{p}}=0.09 \frac{\beta}{\beta+1} \Delta_{\varphi}$, where $\beta$ is the beam intensity ratio between the local oscillator and the probe.

where $T_{2}$ is the excitonic free induction decay (FID) time [16]. Because of the wavelength dependence of $\psi(\omega)$, the quadrature condition [17] is achieved by selecting the operating frequency $\omega_{q}$ at which $\psi(\omega)$ $+\varphi_{P}=0$ or $\pi$, and the homodyne signals depend linearly on the phase modulation. Figure 1 shows the wavelength dependence of two-wave mixing and homodyne signals from a PRQW device measured with a temperature-tunable diode laser (Mellos Griot). At the quadrature frequency $\omega_{q}$, the two-wave mixing signal crosses zero and the homodyne signal reaches its maximum value.

In adaptive spinning-disk interferometry, the probe beam has intensity noise caused by diffraction from the spatial variations in the disk, which is typically much stronger than the phase noise in both the transmission and the reflection mode. These phenomenona can be explained by a simple model from dynamic scattering [18]. The heterogeneous features in the disk substrate, or microscopic roughness features on the surface, scatter light in a manner similar to random particles. The field after scattering can be written as

$$
\tilde{E}=\tilde{E}_{0}+\sum_{i=1}^{N} \delta \tilde{E}_{i}
$$

where $\tilde{E}_{0}$ is the average field, $\delta \tilde{E}_{i}$ is the field contribution from the $i$ th particle, and $N$ is the total number of particles illuminated in the beam. The intensity noise is

$$
\delta P=\left[\tilde{E}_{0} \times\left(\sum_{i=1}^{N} \delta \tilde{E}_{i}\right)^{*}+\sum_{i, j=1}^{N} \delta \tilde{E}_{i} \times \delta \tilde{E}_{j}^{*}+\text { c.c. }\right]
$$

and the phase noise is approximately

$$
|\delta \varphi| \approx\left|\sum_{i=1}^{N} \delta \tilde{E}_{i} \times\left(\tilde{E}_{0}\right)^{-1}\right| .
$$

Because of the random distribution of particles, when $N$ is large (i.e., the domain size of heterogeneities (or 
roughness) is much smaller than the beam size) the fluctuating terms average to

$$
\begin{aligned}
& \frac{\tilde{E}_{0}}{\left|\tilde{E}_{0}\right|} \mathrm{g}\left(\sum_{i=1}^{N} \delta \tilde{E}_{i}\right) \rightarrow \sqrt{N} \sigma_{\delta E} \\
& \sum_{i, j}^{N} \delta \tilde{E}_{i} \mathrm{~g} \delta \tilde{E}_{j}{ }^{*} \rightarrow N{\sigma_{\delta E}}^{2}+\sqrt{N} \sigma_{\delta E}{ }^{2}
\end{aligned}
$$

where $\sigma_{\delta E}$ is the RMS deviation of the scattered field amplitude from each scatterer. This leads to the limiting expressions for intensity and phase noise

$$
\begin{gathered}
\delta P \rightarrow N \sigma_{\delta E}^{2} \\
|\delta \varphi| \rightarrow \sqrt{N} \frac{\sigma_{\delta E}}{E_{0}} .
\end{gathered}
$$

In these equations the intensity noise originates from mixing of the scattered field with itself, while the phase noise only affects the mixing of the scattered field with a local oscillator. Because the scattered field is the sum of a large number random scatterers, noise from homodyne mixing measured on the local oscillator is lower than the noise on the transmitted intensity. In this discussion of adaptive interferometry, the two-wave mixing signal is designated as the homodyne signal because the phase is locked between the signal wave and the local oscillator. This terminology is somewhat different than the terminology typically used in dynamic light scattering [18].

Extracting small phase modulation in the presence of large intensity modulation is difficult for conventional interferometry because the probe and the local oscillator are directly mixed and the intensity noise on the probe beam dominates. However, the adaptive interferometer has separate output ports for the probe beam and local oscillator, which makes it possible to detect the molecular signal in the local oscillator that is free of intensity modulation. Under the condition of quadrature, the transmitted local oscillator is influenced only by the molecular phase modulation and by the phase noise in the probe beam. The transmitted local oscillator, after mixing with the probe wave, is

$$
\begin{aligned}
P_{l}^{\prime} & \propto P_{l}^{0} \pm 2 \sqrt{\eta\left(\omega_{q}\right) P_{l}^{0}\left[P_{p}^{0}+\delta P_{p}(t)^{0}\right]} \sin [\varphi(t)] \\
& \approx P_{l}^{0} \pm 2 \sqrt{\eta\left(\omega_{q}\right) P_{l}^{0} P_{p}^{0}} \varphi(t)+O\left[\varphi(t) \delta P_{p}(t)^{0}\right]
\end{aligned}
$$

neglecting a common overall absorption of the beams in the PRQW, where $\delta P_{p}(t)^{0}$ is the intensity noise in the probe beam, $\varphi(t)$ is the phase modulation, and $\eta$ is the diffraction efficiency. Detection on the local oscillator is sensitive to the phase modulation, but free of intensity modulation to lowest order. By tuning to the quadrature condition, homodyne signals can remain linearly sensitive to the phase modulation while intensity modulation is suppressed. An adaptive interferometer that detects on the local oscillator under the quadrature condition is essentially an AM-free detector similar to FM radio.

The optical thickness change $\Delta(n d)$ detected from a thin layer can be either positive (dielectric, $n>1$, $\Delta(n d)=(n-1) d>0)$ or negative (metal, $n<1$, $\Delta(n d)=(n-1) d<0)$. Intensity-free phase sensitive homodyne signals can tell not only the magnitude of $\Delta(n d)$, but also the sign of the optical thickness change. As Eq. 2 shows, when the wavelength changes, the excitonic spectral phase [16] changes as

$$
\frac{d \psi(\lambda)}{d \lambda}=\frac{d \psi(\omega)}{d \omega} \frac{d \omega}{d \lambda}=-T_{2} \frac{2 \pi c}{\lambda^{2}}<0 .
$$

The two-wave mixing power varies with the excitonic spectral phase as

$$
\Delta P(\lambda) \propto \cos \left[\psi(\lambda)+\varphi_{P}+\pi / 2\right] .
$$

Figure 1 shows that, near the quadrature condition used in the experiments, two-wave mixing flips from positive to negative as wavelength increases, which means that the quadrature wavelength satisfies

$$
\psi\left(\lambda_{q}\right)+\varphi_{P}=\pi .
$$

At this quadrature, the homodyne signal from a phase modulation $\varphi(t)$ is proportional to the cosine of the total phase, given by

$$
\delta P(t) \propto \cos \left[\psi\left(\lambda_{q}\right)+\varphi_{P}+\pi / 2+\varphi(t)\right] \propto \varphi(t) .
$$

Thus the homodyne signal is in phase with the phase modulation, which means that higher (lower) voltage corresponds to longer (shorter) travel in optical thickness.

\section{Optical System}

The system diagram for adaptive spinning-disk interferometry is shown in Fig. 2. The system uses a tunable external cavity diode laser (EOSI 2010), with the wavelength tuned to quadrature near $835 \mathrm{~nm}$. The system can perform both transmission detection of patterns printed on glass disks or reflective detection of patterns on a dielectric reflective disk. Diagrams of two types of disk readers (BioCD Readers) are shown in the bottom of Fig. 2. After mixing the probe beam and the local oscillator in the PRQW device, homodyne signals are read out by an amplified avalanche photodiode (APD1, Hamamatsu C5460) centered on the local oscillator beam and measured by either a spectrum analyzer or an oscilloscope. To calibrate the system, a $5 \mathrm{MHz}$ resonant phase modulator (PM, Newfocus 4001) is located in the probe beam before the disk and the homodyne signal is measured with known phase modulation amplitudes. 


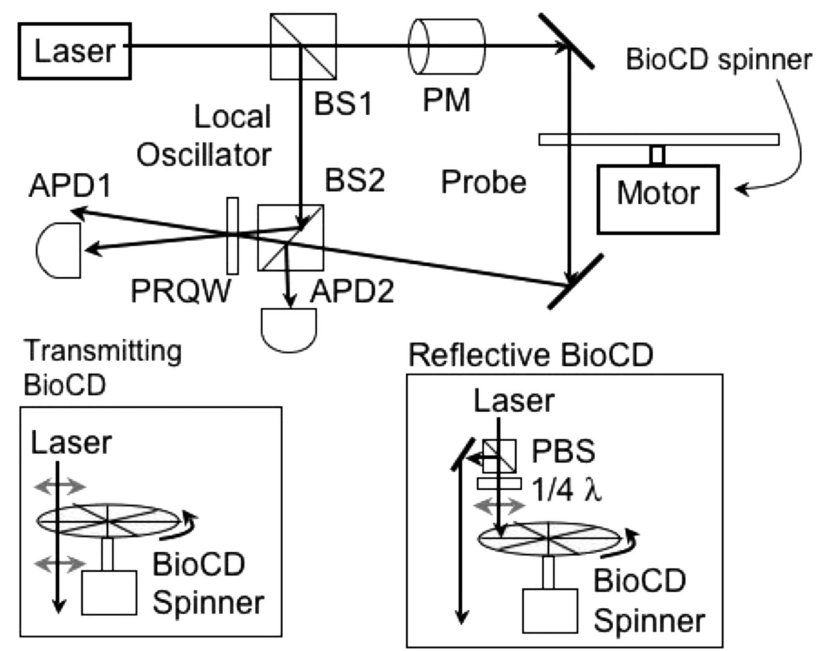

Fig. 2. Diagram of the adaptive spinning-disk interferometry system. The electro-optic phase modulator (PM) provides calibration, while the disk and APD1 provide protein detection on the spinning disk. Intensities of the probe beam are monitored by APD2. Transmitting BioCDs are read by a focused probe beam and a collimating lens after the disk (Lower left). Reflective BioCDs are read by the probe beam through a polarized beamsplitter (PBS), a $1 / 4 \lambda$ wave plate and the focusing lens (Lower right). The reflected probe beam is collimated by the same lens and sent to the PRQW device by the polarized beam splitter.

The spatially periodic patterns of biomolecules immobilized on a spinning disk impart a periodic phase modulation on the probe laser beam. Detecting biomolecules as a spinning phase pattern has two advantages. First, biomolecules are detected by measuring signals at a high frequency, which avoids $1 / f$ noise and allows narrowband detection based on repetitive sampling. Second, the phase signal measured by the system is the optical thickness difference between the molecular spoke pattern and a reference surface. In an assay application, the molecular spoke can be a recognition molecule like an antibody that recognizes and binds a specific molecule in a sample. The reference surface can carry an inert molecule or an antibody that is specific to a different target protein. Nonspecific antibody-protein binding (or cross-reactivity) will be common to both surfaces and is therefore canceled out, representing a form of molecular common-mode rejection.

The phase modulation scheme of adaptive spinningdisk interferometry is shown in Fig. 3. The disk is a 4 "-diameter glass disk with biomolecules printed in a 1024-wedged-spoke pattern, in which the spoke width increases linearly with radius to maintain a 50/50 duty cycle. The probe beam has a focal size of $30-\mu \mathrm{m}$ full-width half-maximum. The spinning disk acts as a phase modulator with a modulation depth that is linear in the optical thickness relief of the biomolecule pattern. Detection is performed on a circular track (illustrated as the black ring in Fig. 3) with radii that are selected by moving the spinner with a computercontrolled linear stage. In experiments, neighboring tracks are often grouped into a single band, covering

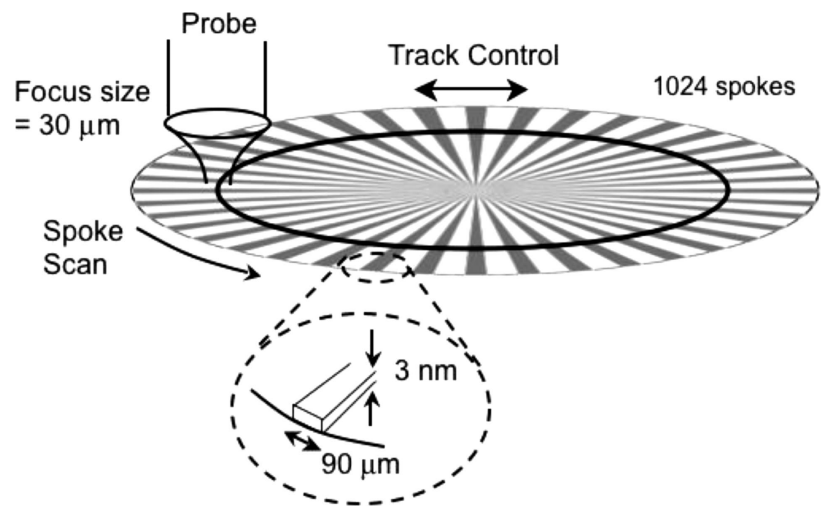

Fig. 3. Adaptive spinning-disk and probe geometry converts a periodic pattern of printed protein on a spinning disk into a highfrequency phase modulation. The disk is printed with 1024 protein spokes with widths increasing with radius to maintain a 50/50 duty cycle. At a radius of $30 \mathrm{~mm}$ the spoke width is 90 microns. The printed protein spoke heights are approximately $3 \mathrm{~nm}$ thick.

typically a several-mm annular width on the disk. Two indexing methods are used to track spoke patterns. Transmission disks have 1-mm-wide gold-patterned synchronization bands on the outer rim providing 1st to 16 th harmonics of the rotation frequency, plus a gold 1024-element band as a carrier-frequency monitor; a secondary laser beam is focused through one of these patterned gold bands as a synchronization signal. When reading reflective disks, an improved disk spinner (Lincoln Lasers) that has built-in digital indexing removes the need for the tracking bands.

\section{System Calibration}

A series of calibration experiments were carried out to demonstrate that adaptive spinning-disk interferometry is capable of scanning nanometer-thick patterns to determine their optical thickness relative to air with high precision despite the presence of intensity modulation and noise. These calibration results validate the latter biomolecule detection application.

\section{A. Calibration Standard Disk}

To calibrate the system, two methods were used: (1) a calibration disk for daily system testing; and (2) an electro-optic phase modulator in the system to calibrate the response of the homodyne signal to a known phase modulation in situ.

The calibration disk is $0.5-\mathrm{mm}$ thick fused silica with marks milled by a 1 min ion beam milling (estimated15-nm deep by Ion Beam Milling, Inc.), which is used for phase modulation in transmission when the disk spins. The disk has numerous synchronization tracks in the outer radii. In the inner radii there is a 1024-spoke pattern that is divided into several circular bands. There is one blank band for the study of system noise floors, and two bands fully covered with the 1024-element pattern. In addition, there are 10 bands in which spoke patterns are turned on and off around the track with modulation frequencies of 1 per track to 16 per track to form a low-frequency 


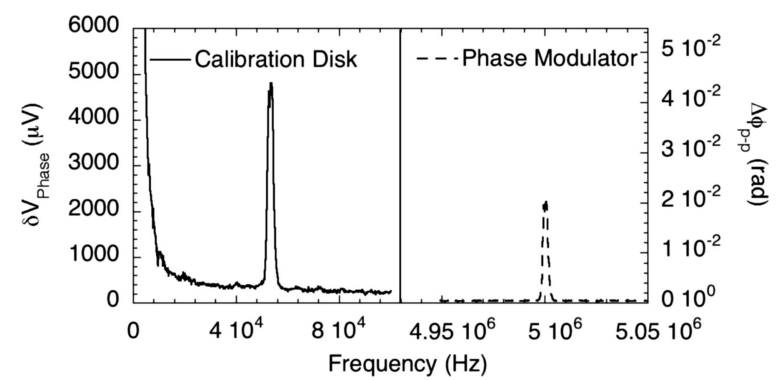

Fig. 4. Homodyne spectra of the full track on the glass calibration disk (solid line) and the phase modulator with $\Delta \varphi_{p p}=2.1$ $\times 10^{-2} \mathrm{rad}$ (dashed line). The left $y$-axis is amplitude, and the right $y$-axis is equivalent phase modulation. The glass milling marks generate phase modulations $\Delta \varphi_{p p}=4.4 \times 10^{-2} \mathrm{rad}(5.8 \mathrm{~nm}$ at $\lambda=$ $835 \mathrm{~nm}$ ). The noise level of the calibration track is equivalent to $\Delta \varphi_{p p-r m s}=3 \times 10^{-3} \mathrm{rad}(0.4 \mathrm{~nm}$ at $\lambda=835 \mathrm{~nm})$.

spatial modulation envelope that multiplies the highfrequency carrier frequency.

The calibration disk is used to verify the frequency response of the PRQW device. PRQW devices selfcompensate low-frequency phase modulation, and have a high-pass frequency response for adaptive homodyne detection. Previous studies [15] showed that the compensation rate of a typical PRQW device is $70 \mathrm{kHz}$ with a similar laser intensity $\left(<3 \mathrm{~mW} / \mathrm{cm}^{2}\right)$. Compensation rates vary between devices made from different fabrication batches. Homodyne signal spectra from the 1024-element track on the calibration disk were taken at spinning speeds from 20 to 70 rotations per second. The first, second, and third harmonic peaks of the homodyne signals, which extend from $20 \mathrm{kHz}$ to $215 \mathrm{kHz}$, have the same amplitude regardless of the spinning speed, which proves that the compensation rate of the PRQW device used in the system is below $20 \mathrm{kHz}$. Thus, homodyne signals from the calibration disk can be compared against the homodyne signal generated by the phase modulator to measure the milling depth. Figure 4 shows the homodyne spectrum from a track fully covered with the 1024-element pattern and compared to the spectrum from the phase modulator, both taken with a spectrum analyzer set at a $3-\mathrm{kHz}$ bandwidth. The phase modulator is driven by a $5 \mathrm{MHz} \mathrm{AC}$ voltage with $V_{p p}$ equal to $40 \mathrm{mV}$, which is equivalent to a peak-to-peak phase modulation with $\Delta \varphi_{p p}=2.1$ $\times 10^{-2} \mathrm{rad}$, or a peak-to-peak optical thickness change $\Delta n d=2.8 \mathrm{~nm}$ at $835 \mathrm{~nm}$. By comparing these two homodyne signals, the milled marks are measured to be $\Delta(n d)=(n-1) \Delta d=5.8 \mathrm{~nm}$ in optical thickness, or $\Delta d=12 \mathrm{~nm}$ in physical thickness (close to the estimated thickness of $15 \mathrm{~nm}$ from the ion milling).

\section{B. Sensitivity and Noise}

Noise from a spinning disk is caused by disk heterogeneity. In the case of transmission, the heterogeneity is a combination of surface roughness and density variation in the glass. In the case of reflection it is primarily from surface roughness. The noise spec-

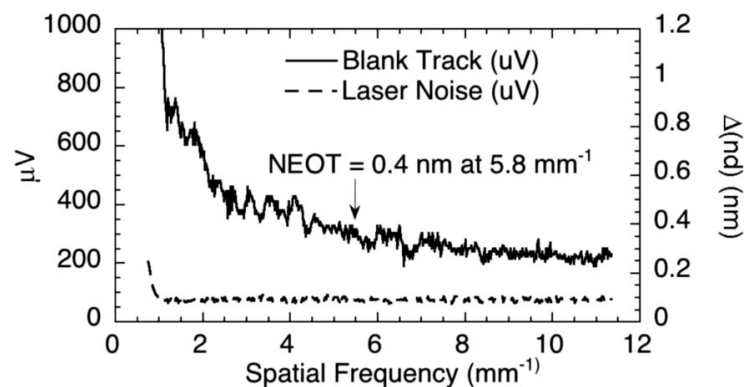

Fig. 5. Homodyne signal from the blank track showing phase noise. Spectra were taken with a spectrum analyzer at a radius equal to $29 \mathrm{~mm}$. The disk was spun at $50 \mathrm{~Hz}$ with $3-\mathrm{kHz}$ acquisition bandwidth, which is equivalent to $0.3 \mathrm{~mm}^{-1}$ in spatial frequency. Left $Y$-axis is the voltage, and right $Y$-axis is the noise equivalent optical thickness (NEOT) calibrated by the phase modulator.

trum of spinning-disk interferometry as a function of temporal frequency corresponds directly to the spatial frequency distribution of the heterogeneity or roughness. The spectrum of an intensity-free homodyne signal from a blank track (no milling marks) of the transmission calibration disk is shown in Fig. 5 as a function of spatial frequency, which is calculated from the temporal frequency by taking

$$
f_{\text {spatial }}=\frac{f_{t}}{2 \pi R f_{\text {spin }}}
$$

where $R$ is the radius of the track. Compared with the laser noise spectrum, the spectrum of the blank track is dominated by a homodyne signal from the transmitted phase noise of the spinning glass disk. The spatial distribution of the phase noise is consistent with a white noise spectrum filtered by a low pass filter (the spatial Fourier transform of the Gaussian beam). Unlike stochastic laser noise, the phase noise associated with spatial structure is repeatable on successive acquisitions, and thus cannot be decreased by averaging.

The signal-to-noise ratio (SNR) of the system is defined by

$$
\bar{S}=\frac{\left(\delta P_{L}^{\prime}\right)^{2}}{N_{L}{ }^{2}+N_{P}{ }^{2}}=\left(\frac{\delta d}{\mathrm{NEOT}}\right)^{2}
$$

where $N_{L}$ and $N_{P}$ are noise amplitudes contributed by the local oscillator and by the phase noise from the disk, respectively. The sensitivity of the system is determined by its noise equivalent optical thickness (NEOT), which is given by

$$
\mathrm{NEOT}=\delta d \times \frac{V_{\text {Homo }}}{V_{\text {noise }}}
$$

where $V_{\text {Homo }}$ is the homodyne voltage from a phase modulation equal to $\delta d$ in optical thickness, and $V_{\text {noise }}$ is the noise voltage. The blank-track spectrum was 
taken at the radius $R=28 \mathrm{~mm}$ with a $0.3 \mathrm{~mm}^{-1}$ spatial bandwidth (corresponding to a $3 \mathrm{kHz}$ bandwidth on the spectrum analyzer) and calibrated with the phase modulator signal (Fig. 5, right $y$-axis). The temporal carrier frequency for the 1024-spoke pattern at the spin frequency $f_{\mathrm{s}}=50 \mathrm{~Hz}$ is $f_{\mathrm{t}}$ $=51.2 \mathrm{KHz}$, and the spatial frequency is $f_{\mathrm{sp}}=$ $5.8 \mathrm{~mm}^{-1}$. At this spatial frequency of $5.8 \mathrm{~mm}^{-1}$ the NEOT of a spinning glass disk in transmission is $0.4 \mathrm{~nm}$, and the NEOT of the laser noise is $0.1 \mathrm{~nm}$ under equal probe beam and local oscillator intensities with a detected $3 \mu \mathrm{W}$ optical power and a $0.3 \mathrm{~mm}^{-1}$ spatial bandwidth.

In practice, the sensitivity is also affected by the mechanical stability of the spinner and the flatness of the disk. Uneven disk surfaces and disk wobble during spinning could cause a high-frequency Doppler shift in the probe beam, and subsequently fringe wash-out in the PRQW device. For example, to cause a $20 \mathrm{kHz}$ Doppler shift, the optical path needs to drift at a speed of $1.7 \mathrm{~mm} / \mathrm{s}$. Such drift is unlikely to happen in the transmission mode, since path length drifts come from uneven disk thickness (typically $\pm 25 \mu \mathrm{m}$ across the whole disk), which can only produce a $0.1 \mathrm{~mm} / \mathrm{s}$ drift in the worst case. However, in the reflective mode, disk wobble could cause big drifts if the spinner lacks stability. Thus, a high-precision spinner (Lincoln Laser) was used in the reflected mode so that the interference fringes remain visible through a video camera even when the disk is spinning. In both transmission and reflection modes, the calibration signal from the EO modulator dropped less than $1 \mathrm{~dB}$ in amplitude when the disk was spinning. This provides strong evidence that the system compensates most of the optical path drift. To take this $1 \mathrm{~dB}$ decrease into account, all phase calibration signals were taken with the disk spinning.

\section{Optical Thickness Measurement of Thin Gold Layers}

The advantage of intensity-free detection on the local oscillator is the ability to measure the optical thickness of thin layers despite the presence of reflection or absorption that cause large intensity modulations. The performance was first tested with thin gold layers. Figure 6 plots the transmission and homodyne traces by spinning a $15-\mathrm{nm}$ thick gold spoke pattern (thinner than an extinction depth) evaporated on a glass disk. The pattern was made by lithography and thermal evaporation at a deposition rate of $1-2 \AA / \mathrm{s}$. In the transmission trace, the minimum of the modulation corresponds to the gold spokes, which is less transparent than the glass substrate. At the same time, the phase-sensitive homodyne signal from the gold spokes also gives a lower value, which means that the gold spokes causes a negative phase shift $\Delta(n d)<0$. With the phase modulator as the calibration, the optical thickness of the 15-nm gold layer was measured to be $n d=d-|\Delta n d|=15-8=7 \mathrm{~nm}$, with a refractive index of $n=0.5$. The dielectric constant of a nonannealed gold film was reported to be $\varepsilon=-31+5.1 i$ at $835 \mathrm{~nm}$ (extracted from data

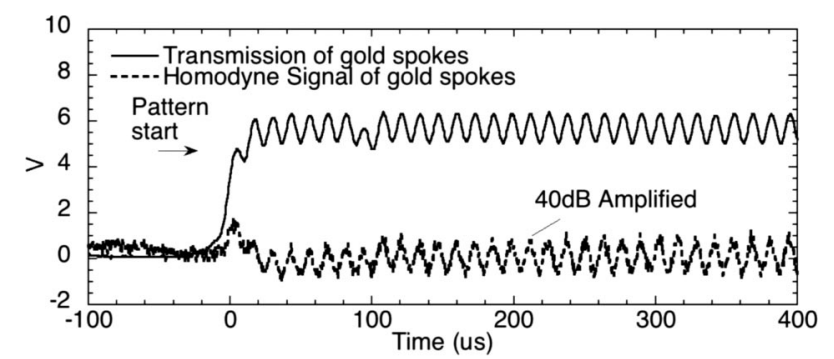

Fig. 6. Single-shot oscilloscope traces of intensity and phase signals from a spinning 15-nm thick gold spoke pattern. Acquisitions were triggered by an opaque edge on the disk. The intensity modulation and homodyne signals from phase modulations were inphase. The gold spokes were spun at $5000 \mathrm{rpm}(\sim 80 \mathrm{~Hz})$. The beam intensity ratio $\beta$ was equal to 1 . Homodyne signal was calibrated to be $\delta V_{P}=0.27 \Delta_{\varphi}$ by the phase modulator. The corresponding optical thickness change $\Delta(n d)$ was $8 \mathrm{~nm}$.

plots in Ref. 19), which gives a refractive index $n$ $=\operatorname{real}(\sqrt{\varepsilon})=0.5$ that matches our results. This demonstrates the versatility and robustness of intensityfree detection.

\section{Adaptive Optical BioCD}

The surface profile sensitivity that is achieved with spinning-disk adaptive interferometry opens many avenues for surface metrology applications. In our first application of this technique [2], we investigated the detection of printed antibody patterns on glass disks. In this section, we expand on that work and then extend it to higher sensitivities by operating in reflection on a dielectric stack designed to have a field antinode at the surface and hence optimized fieldmolecule interaction.

\section{A. Biomolecule Pattern Printing}

Printing a biomolecular pattern on the BioCD is the first step to biosensing. The process consists of two steps: surface activation and biomolecule patterning. Two surface chemistries were used for surface activation. One is grafting of chlorodimethyloctadecylsilane [20] onto the silica surface, which creates an adhesive coating that attaches biomolecules by physical adsorption. This procedure is simple but can suffer from molecule wash-off during experiments due to the unstable nature of physical adsorption. A more stable approach is a biotin-avidin-based system, in which the silica surface is covalently coated with a biotin-labeled polysuccinimide (PSI) coating [21] Avidin, a protein that binds to biotin with high affinity, serves as the link between the disk coating and the biotin-labeled biomolecules that are to be immobilized. Three alternating printing methods were used in our biomolecule patterning: microfluidic network printing [22], photolithography, and a soft "ink" gel stamp method [23].

Both the microfluidic network printing and the "ink" gel method start with a master wafer that has a 25 - $\mu \mathrm{m}$-thick SU-8 photoresist in the 1024-spoke pattern. In the microfluidic method, a polydimethylsiloxane (PDMS) stamp is molded against the master 
wafer. After curing, the stamp is removed from the master, treated for $3 \sim 5$ min with oxygen plasma, and then sealed with the disk to form micro-channels. To print, $100 \mu \mathrm{g} / \mathrm{ml}$ protein solution is added through a central reservoir and initially fills the channels by capillary force. Incubations last $30 \mathrm{~min}$, during which time the disk is spun at $200 \mathrm{rpm}$, and the solution flows by centrifugal force. After the incubation, channels are flushed with buffer solution by spinning at $400 \mathrm{rpm}$.

The gel-stamp method is a modification of the regular micro contact printing method [23], which uses a polydimethylsiloxane (PDMS) stamp. We opted for a soft, wet gel stamp because PDMS stamps produce uneven printing over our large disks (100 mm diameter). A $12 \%$ acrylamide gel solution containing 50 $\mu \mathrm{g} / \mathrm{ml}$ of protein (antibody or antigen) is poured into a gel-casting chamber containing the master wafer. After curing, the chamber is opened, and the gel stamp is removed from the master wafer. With the stamp facing up, a disk is carefully laid onto the stamp and let to stand for $15 \mathrm{~min}$ At the end, the disk is washed with an excess of water to remove extra proteins.

The photolithography method only works with the PSI chemistry which can withstand the harsh lithography procedure. First, standard photolithography is performed on PSI-coated disks. Photoresistpatterned disks are incubated in avidin solution for $10 \mathrm{~min}$, cleaned with water, and the photoresist is stripped off by acetone. The photolithography method gives the best accuracy and resolution. However, the microfluidic and "ink" gel approaches produced relatively uniform patterns at low cost with ease and were used in most of our experiments.

\section{B. Optical Thickness and Refractive Index of a Protein Monolayer}

The homodyne spectrum of a circular track of a FITC fluorescein-labeled BSA (Sigma Aldrich) pattern is shown in Fig. 7(a). The BSA was printed by gel stamping on a glass disk activated with the physical adsorption coating. The inset in Fig. 7(a) is the fluorescence photo of the BSA pattern. The optical thickness change from the protein pattern $\Delta(n d)$ was measured to be $0.6 \mathrm{~nm}$ calibrated by the electro-optic modulator. To verify the properties of the printed protein, atomic force microscopy was used to scan the surface relief of the pattern. Fig. 7(b) shows a 10 $\times 10 \mu \mathrm{m}$ scan of the edge of a BSA-FITC spoke, which shows that the BSA region was $2.5 \mathrm{~nm}$ higher on average than the land level, while both had a roughness of $0.5 \mathrm{~nm}$. These results show that a relatively uniform monolayer coverage of BSA-FITC was printed with a thickness of $d=2.5 \pm 0.7 \mathrm{~nm}$. The optical thickness change $\Delta(n d)=(n-1) d=$ $0.6 \mathrm{~nm}$ for BSA-FITC is used to estimate the refractive index of the BSA monolayer layer to be $n=$ $1.2 \pm 0.1$.

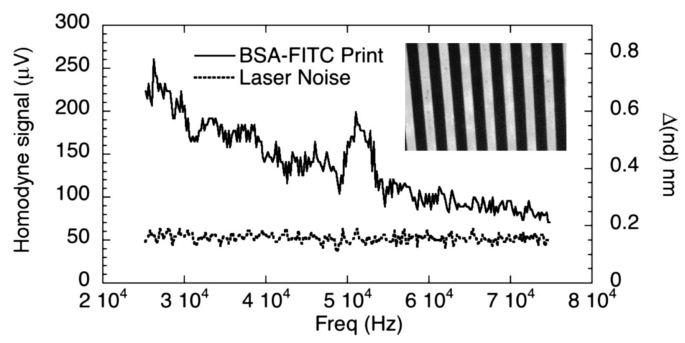

(a)

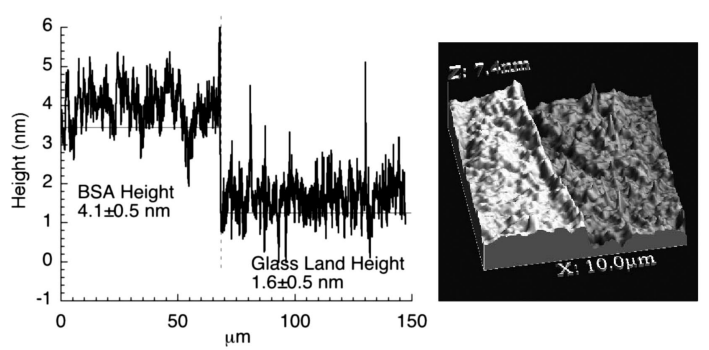

(b)

Fig. 7. Optical thickness of a protein monolayer. Redrawn from Ref. 2. (a) Homodyne spectrum of BSA-FITC printed by gel stamping. The left $Y$-axis is the amplitude and the right $Y$-axis is the equivalent optical thinness change calibrated by the phase modulator. The optical thickness of the protein pattern was measured to be $0.6 \mathrm{~nm}$ corresponding to a physical thickness of $2.5 \mathrm{~nm}$. Insert: fluorescence photo of the pattern. (b) BSA spoke edge profile scanned by atomic force microscope: profile of a BSA edge on glass region (left) and 3D map (right).

\section{Immunoassay on an AO-BioCD}

To demonstrate the AO-BioCD as a biosensor, we implemented an immunoassay in which printed antigen binds specific antibodies out of solution, called a reverse immunoassay. The molecular recognition is detected as an optical thickness change in the protein pattern after the reaction. Single and two-analyte assay experiments were performed in transmission with protein immobilized by the physical adsorption protocol described earlier. In each experiment, about 150 tracks of the protein pattern were acquired at radii separated by a radial pitch of $0.1 \mathrm{~mm}$. Tracks were grouped into bands, on which sample incubations were carried out in multiple steps on selected bands. Antigens and antibodies used in experiments were obtained from Sigma Aldrich and diluted to 200 $\mu \mathrm{g} / \mathrm{ml}$ in phosphate buffer (10 mM PH 7.4). Incubations lasted for $15 \sim 30 \mathrm{~min}$, followed by a gentle wash with $0.05 \%$ Tween 20 and then distilled water that was blown dry. Disk optical thicknesses were re-measured after each step on the dried disk.

Figure 8 is a graphical display of the signal spectra taken during a single analyte (Fig. 8(a)) and a twoanalyte (Fig. 8(b)) experiment. Each frame in the figure is a $2 \mathrm{D}$ map of tracks, with radii as the $\mathrm{Y}$-axis and frequency as the $\mathrm{X}$-axis. The vertical bright stripe in each frame is the carrier frequency centered at $51.2 \mathrm{kHz}$. The brightness of the carrier frequency is related to the contrast of the spoke pattern. As proteins are added to the spokes and the spokes get higher (gain mass) relative to the land level, the brightness increases. As proteins are added to the 

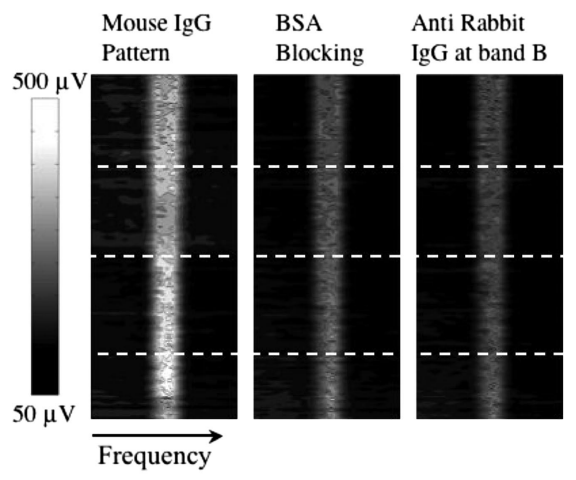

Anti Mouse IgG
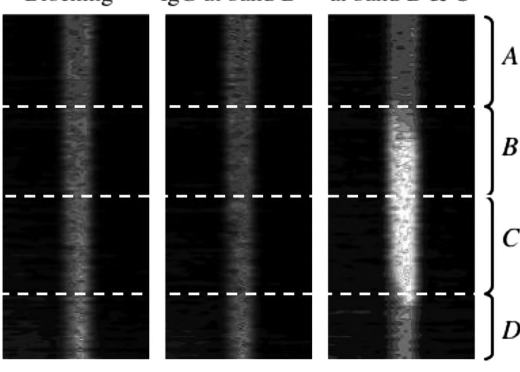

(a)

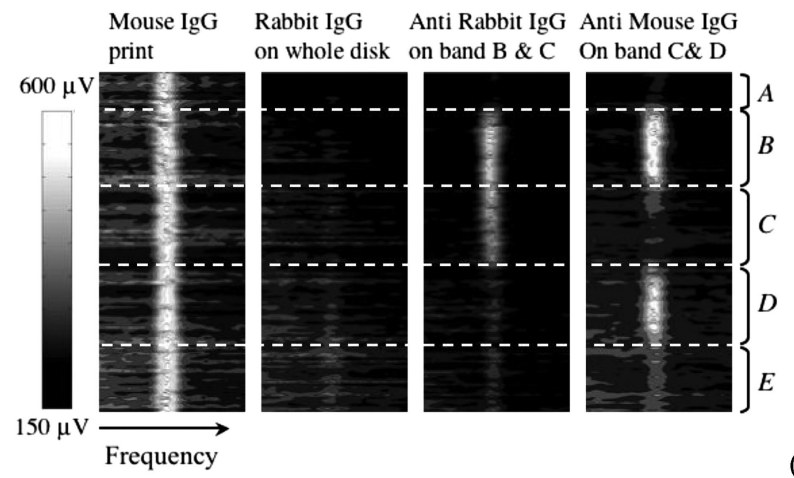

(b)

Fig. 8. Immunoassays on the AO BioCD. Homodyne spectra were taken on tracks with $0.1-\mathrm{mm}$ radius steps. Amplitudes $(\mu \mathrm{V})$ of spectra are plotted in a $2 \mathrm{D}$ gray scale map with the $x$-axis representing frequency (with the protein center-frequency at $51.2 \mathrm{kHz}$ with a $3 \mathrm{kHz}$ detection bandwidth) and the y-axis representing the radius. The incubations used $200 \mu \mathrm{g} / \mathrm{ml}$ of analyte in buffer solution with incubation times of $20 \mathrm{~min}$. (a) Single-analyte experiment to detect Mouse IgG and Anti Mouse binding. Frame 1: Microfluidic-printed pattern of mouse IgG. Frame 2: after BSA incubation. Frame 3: Band B was incubated with nonspecific antibody Anti-Rabbit IgG. Frame 4: after both bands B and C were exposed to specific antibody antimouse IgG. Bands A and D were reference bands that were not incubated. (b) Two-analyte experiment to detect mouse IgG anti-Mouse IgG binding and rabbit IgG antirabbit IgG binding. Frame 1: printed mouse IgG. Frame 2: after global incubation with rabbit IgG. Frame 3: Bands B and C were exposed to antirabbit IgG. Frame 4: Bands C and D were exposed to antimouse IgG. Bands $\mathrm{A}$ and $\mathrm{E}$ were reference bands.

land between the spokes, the signal at the carrier frequency decreases. The different annular bands (denoted on the right of the frames) are exposed to reagents in sequences depending on the 1-analyte or 2 -analyte protocols.

The single-analyte experiment had four steps (frames from left to right). The whole disk area was divided into 4 bands $(\mathrm{A} \sim \mathrm{D})$. First, mouse IgG antigen was printed in a 1024-spoke pattern on the disk by the microfluidic method. Spectra from the mouse IgG pattern have uniform peaks at the pattern carrier frequency, indicating a uniform print (frame 1). Then the entire disk was incubated with $200 \mu \mathrm{g} / \mathrm{ml}$ BSA as the blocking agent. The signal at the carrier frequency in frame 2 decreased because the blocking agent filled in the land and decreased the pattern contrast. In the third step, band B was incubated with a nonspecific antibody, antirabbit IgG antibody. After the nonspecific incubation of band B, signals in frame 3 from band B showed no difference compared to the rest of the three bands. This demonstrates that either there was no binding of the nontarget analyte, or the nontarget analyte binds equally to the land and the protein spoke. Finally, bands B and C were incubated with the specific antibody, antimouse IgG. After the specific incubation of bands $\mathrm{B}$ and $\mathrm{C}$, in frame 4, signals from both $\mathrm{B}$ and $\mathrm{C}$ showed approximately equal increases at the carrier frequency. This result demonstrates that the printed antigen is successfully recognized by the target antibody in solution, and also shows that the prior exposure to nonspecific reagents did not affect the specific molecular recognition.

The two-analyte assay experiment had two targets, targeting mouse IgG to antimouse IgG and rabbit IgG to antirabbit IgG. The two reactions served as the nonspecific reference for each other. The disk was divided into five annular bands. First, mouse IgG was printed on the disk by the microfluidic method. Frame 1 shows uniform signals from the printed mouse IgG patterns. In the second step, rabbit IgG antigen at $200 \mu \mathrm{g} / \mathrm{ml}$ was used as the free-surface saturating reagent (instead of the BSA used in the 1-analyte protocol). Signals in frame 2 dropped to near the noise level as the land filled in, indicating that the rabbit IgG layer on free surfaces has an optical thickness similar to that of mouse IgG. Note that the entire disk is flat after this last step, but the surface has two functionalized surfaces in alternating spoke patterns: one mouse antigen and the other rabbit antigen.

In the third step, both bands B and C were incubated with antirabbit IgG antibody. Signal peaks reemerged on these same bands $\mathrm{B}$ and $\mathrm{C}$ because the specific binding to rabbit IgG increased the rabbit spokes relative to the mouse spokes in these bands. Finally, both bands C and D were incubated with antimouse IgG. Spectra in frame 4 exhibit two opposite effects. In band D, that was incubated to antibody for the first time, the signal increased because of positive binding by antimouse IgG to the mouse antigen spokes. But in band $\mathrm{C}$ that had been exposed in the previous incubation step, and had already given a positive reading to antirabbit IgG, the second incubation decreases the previously positive signal. This signal cancellation is because the adjacent rabbit and the mouse antigen spokes had both increased in height. In this case, a double positive cancels, which demonstrates that the system detects the phase difference between the pattern and its surrounding land. It also indicates that mouse IgG and rabbit IgG have equally high affinity and specificity in binding to their counterparts.

The results of the 2-analyte procedure from Fig. 8(b) are shown quantitatively in Fig. 9 that traces the effects of each step. The average protein signal is plotted as a function of disk radius for the three successive steps. The vertical arrows on the graphs show how each step either increases or decreases the sig- 


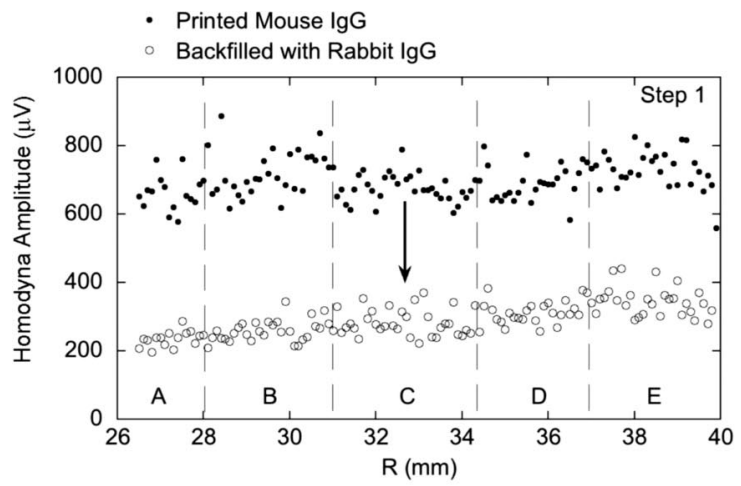

(a)

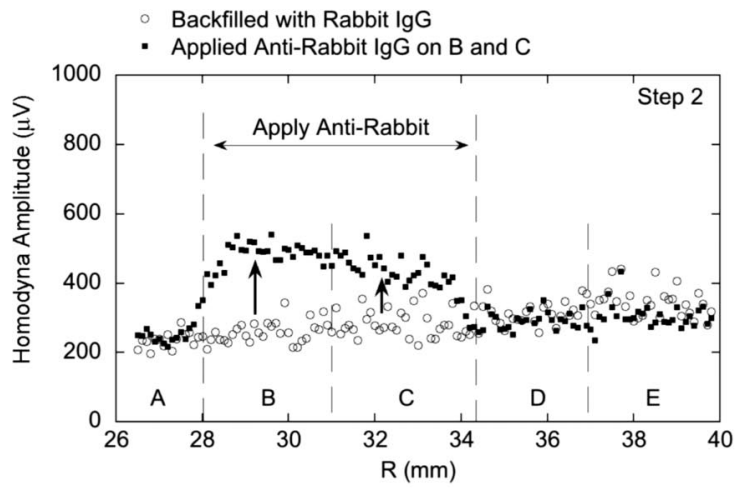

(b)

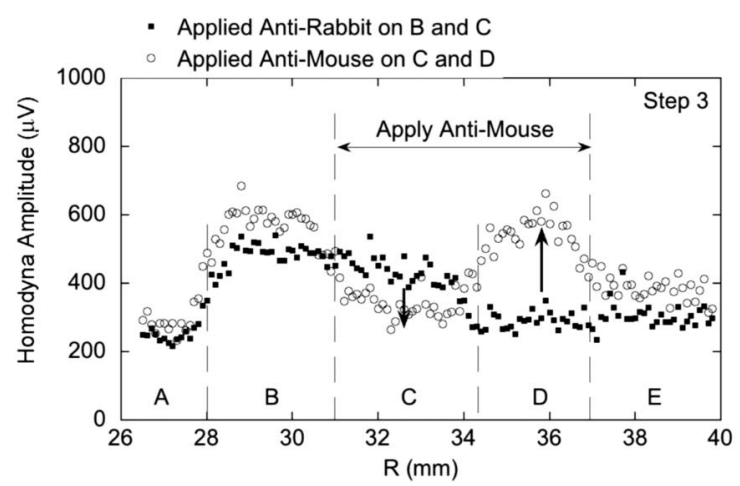

(c)

Fig. 9. Quantitative results of the 2-analyte experiment showing the change in the protein signal as a function of disk radius for the three successive steps in the procedure. In step 1, the disk printed with mouse IgG is backfilled with rabbit IgG. In Step 2, the backfilled disk is incubated with antirabbit antibody on bands B and C. In Step 3, the disk is incubated in bands $\mathrm{C}$ and $\mathrm{D}$ with antimouse antibody.

nal. In Fig. 9(a), the disk printed with mouse IgG (closed circles) is backfilled with rabbit IgG (open circles), and there is a nearly uniform decrease in signal across all bands. In Fig. 9(b), the backfilled disk (open circles) is incubated with antirabbit antibody on bands $\mathrm{B}$ and $\mathrm{C}$, and the signal increases in these bands only (closed circle). In Fig. 9(c), the previously incubated disk (closed circles) is incubated again, but now in bands $\mathrm{C}$ and $\mathrm{D}$ with antimouse antibody (open circle). In band C, that shared both specific incubations, the signal drops, but the signal increases in band D. Both of these effects, the one positive and the other negative, are the result of positive binding to the antimouse antibody.

These two assays, the 1-analyte and the 2-analyte, demonstrate several important principles of immunoassays. First, there is little or no binding that occurs between nontarget antigen-antibody pairs. Second, prior exposure to a nonspecific target does not desensitize the functionalized surface. Third, the BioCD is a platform that can be used for multiple analytes with tag-free direct optical detection. And finally, the technique is phase sensitive, in that two positive bindings on adjacent spokes cancels the amplitude at the carrier frequency.

\section{Reflective AO BioCD: Adaptive Spinning-Disk Topology}

Adaptive spinning-disk interferometry can work both in transmission and reflection. The reflection mode has an advantage over the transmission mode because a reflective surface can have an antinodal field condition with an enhanced optical surface field. Unlike a transmitting surface, which allows the protein layer to be polarized by a traveling field, an antinodal reflective surface places the protein layer at the field maximum of a standing wave. This leads to an increase that is larger than a factor of two for the phase change from the protein layers because of stronger field-dipole interaction. The theoretical phase change of a bioloayer of thickness $d$ and refractive index $n$ on a perfectly reflecting antinodal surface $(r=+1)$ is $\Delta \varphi_{\text {anti-node }}=\frac{4 \pi d}{\lambda}\left(n^{2}-1\right)$ compared with $\Delta \varphi_{\text {trans }}=$ $\frac{2 \pi d}{\lambda}(n-1)$ for transmission.

The homodyne signal from protein printed on a dielectric stack with an antinode surface boundary condition is shown in Fig. 10. The protein signal is from FITC conjugated BSA in a 1024-spoke pattern printed with a gel stamp. The structure of the $98 \%$ reflective dielectric coating at $835 \mathrm{~nm}$ is a sandwich of 20 layers of quarter-wave silicon dioxide $(146.7 \mathrm{~nm}$ $\left.\mathrm{SiO}_{2}\right)$ and tantalum oxide $\left(93.5 \mathrm{~nm} \mathrm{Ta}_{2} \mathrm{O}_{5}\right)$ with a quarter-wave silicon dioxide layer as the cap layer.

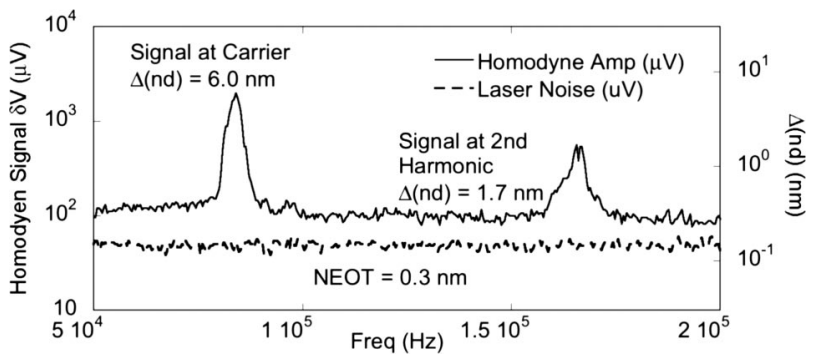

Fig. 10. Homodyne signal spectrum from gel-printed BSA-FITC pattern immobilized on a dielectric antinode reflective disk. The disk was spun at $80 \mathrm{~Hz}$. The left $Y$-axis is the homodyne signal measure by a spectrum analyzer with $3-\mathrm{kHz}$ bandwidth. The right $Y$-axis is the calibrated optical thickness change $\Delta(n d)$ in log scale. The carrier peak has $\Delta(n d)=6.0 \mathrm{~nm}$, and the second harmonic peak has $\Delta(n d)=1.7 \mathrm{~nm}$. The noise-equivalent optical thickness (NEOT) is $0.3 \mathrm{~nm}$ for a detected power of $3 \mu \mathrm{W}$. 


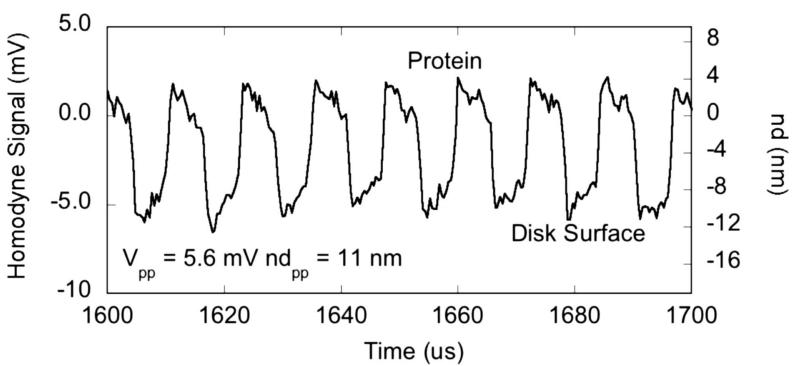

Fig. 11. Homodyne signal trace example of spinning avidin spokes on a reflective disk. Signals are acquired with 32 averages and triggered with the $80-\mathrm{Hz}$ spin-tracking signal from the spinner. Homodyne voltage (left $y$-axis) is converted into optical path length (nd) (right $y$-axis) using the phase modulator calibration.

The noise of a reflective disk comes from a combination of the surface roughness and inhomogeneity within the first several layers. In this case the NEOT is approximately $0.3 \mathrm{~nm}$ after protein printing, which is comparable to the noise in the transmission case. Although the noise levels are about the same, the signal from BSA-FITC is enhanced from $0.6 \mathrm{~nm}$ to $6.0 \mathrm{~nm}$. The second harmonic of the carrier frequency, which had not been detectable in the transmission mode, is evident in the reflection power spectrum. The irregular peak shape of the second harmonic also suggests that with such high signalto-noise ratio, pattern distortion in the gel stamp becomes detectable.

With the NEOT equal to $1 / 20$ of a protein monolayer, specific protein patterns now can be resolved. Fig. 11 is a sample of a homodyne time-domain signal taken from an avidin pattern printed by lithography on the PSI-coated reflective disk. The avidin layer on the antinode reflective surface causes a $10-\mathrm{nm}$ optical path change. A topographic image of the protein pattern was constructed by scanning at different radii.

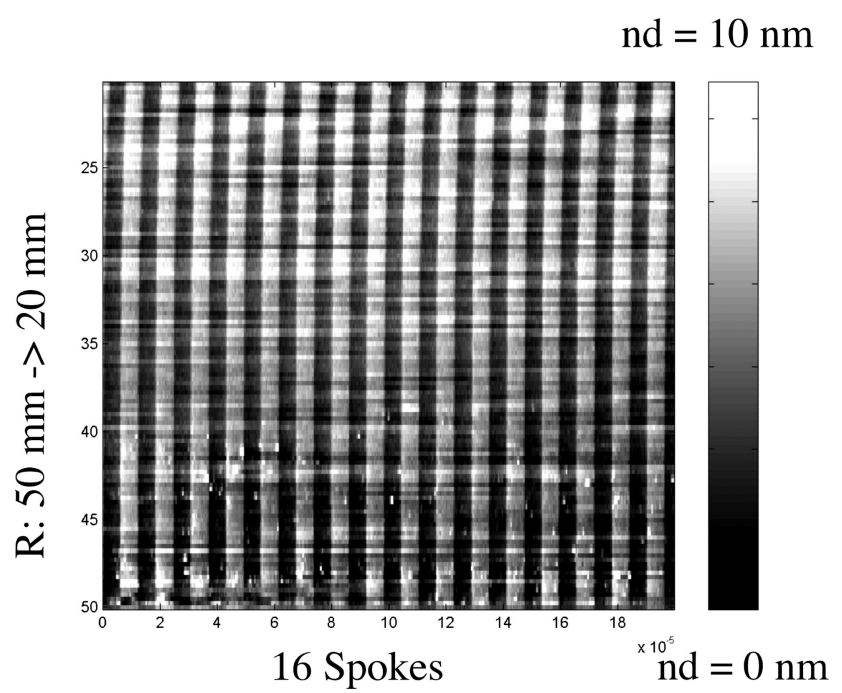

Fig. 12. Topographic map of the avidin-spoke pattern fabricated by photolithography on a PSI coating on a reflective disk. The map covers an area of approximately $1 / 64$ of the disk. The spoke widths are 100 microns. The average protein height was $7 \mathrm{~nm}$.

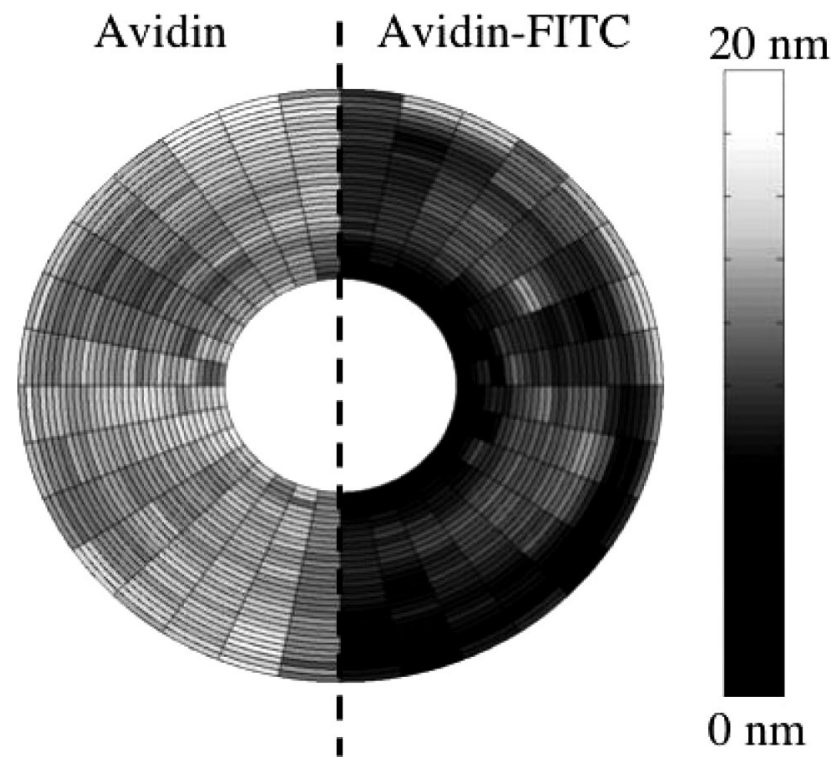

Fig. 13. Whole-disk map of the demodulated protein signal. Half of the disk has regular avidin from egg white (left), and the other half (right) has FITC-conjugated avidin. Average heights of the 960 segments are $10.8 \mathrm{~nm}$ on the left and $7.3 \mathrm{~nm}$ on the right.

Fig. 12 shows the topographic image of the avidin spokes on a part of the disk, in which 16 avidin spokes extend $30-\mathrm{mm}$. A segmented map of $\Delta(n d)$ from avidin spokes on the whole disk is shown in Fig. 13. The area is divided into 30 radial bands, and each band is

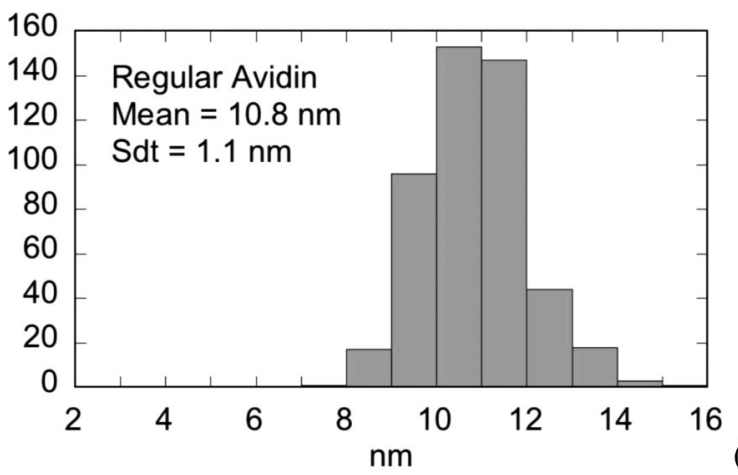

(a)

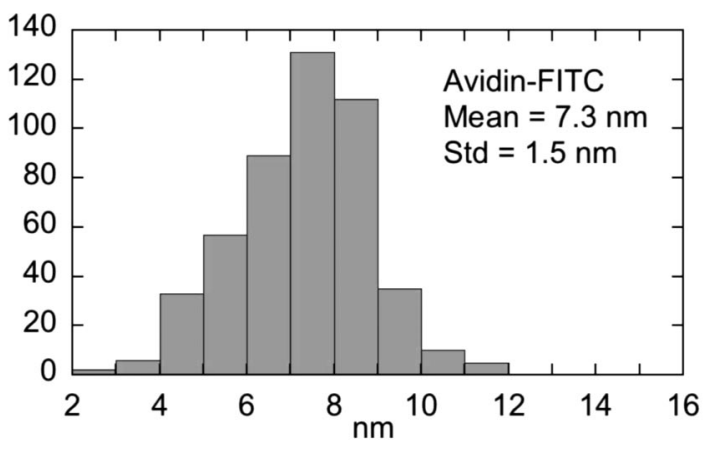

(b)

Fig. 14. Histograms of the average segment heights for the 960 segments of Fig. 13. (a) Left-side segment height from avidin from egg white, (b) right-side segment height from FITC-conjugated avidin. 
divided into 32 angular segments for a total of 960 individual segments with an average area of 2.4 $\times 2.5 \mathrm{~mm}^{2}$. The equivalent spatial bandwidth of the spectrum analyzer is $0.3 \mathrm{~mm}^{-1}$, which is about the inverse of the linear size of each segment. In each segment, the protein signal is analyzed by taking the Fourier transform of oscilloscope traces from that region.

The optical thickness change $\Delta(n d)$ is obtained by calibrating the Fourier power of the signal with the electro-optic phase modulator signal. One half of the disk was printed with spokes of avidin-FITC (Sigma Aldrich) and the other half was printed with regular avidin from egg white (Sigma Aldrich). The map in Fig. 13 shows that the two kinds of avidin spokes had different optical thicknesses. Histograms of the segment heights for the two halves of the disk are shown in Fig. 14. Unconjugated avidin spokes had $\Delta(n d)$ equal to $10.8 \mathrm{~nm}$, which was higher than $\Delta(n d)$ from the conjugated avidin-FITC $(7.3 \mathrm{~nm})$. The standard deviations of the two distributions are 1.1 and $1.5 \mathrm{~nm}$ respectively. The reason for this difference of height is not known. However, we speculate that the avidin conjugated with FITC was less efficient at binding with the biotin on the disk surface.

\section{Conclusion}

In this paper, we have demonstrated that adaptive free-space interferometry is capable of detecting and quantifying thin-layer patterns with subnanometer sensitivity. The detection was carried out in CD-like surface-normal scans, and molecular layers were detected by their optical thicknesses. We studied the cases of transmission through transparent glass disks, and reflection from dielectric stacks with an electric field antinode condition on the surface. The noise-equivalent optical thickness (NEOT) for each case was $0.4 \mathrm{~nm}$ and $0.3 \mathrm{~nm}$, respectively, at a detection bandwidth of $3 \mathrm{kHz}$ and $3 \mu \mathrm{W}$ detected power.

An important feature of our adaptive-optical spinning-disk interferometry system is the detection of phase modulation on the local oscillator. This beam is free of strong intensity effects that arise when the signal beam is transmitted through the spinning disk. As one extreme demonstration of this intensityfree phase detection, we evaporated $15-\mathrm{nm}$ thick spokes of gold (approximately half the extinction depth) on a glass disk. The transmitted signal beam exhibited large intensity modulation because of the high reflectance from the gold surface. However, the local oscillator was free of the intensity modulation, instead exhibiting an intensity modulation proportional to the optical path length of the gold layer. This allowed the estimation of the real part of the gold refractive index to be $n_{r}=0.5$, which agrees with published values. This result, together with the internal calibration provided by the EO modulator, demonstrates the sensitive surface metrology that is possible with this adaptive system.

Based on this surface metrology sensitivity, we implemented immunoassays on the AO-BioCD to detect the molecular recognition and binding of proteins out of solution. The biosensor was demonstrated at a concentration of $200 \mu \mathrm{g} / \mathrm{ml}$, which is well above the saturated range of the chemical kinetics, but illustrates the full dynamic range of the technique. The recognition antigen layer had an effective optical thickness of approximately $0.6 \mathrm{~nm}$ on a transmitting surface. The effective optical thickness was enhanced to $6 \mathrm{~nm}$ by the antinodal reflective disk, whereas the noise level remained in the subnanometer range. Within the focal area of the $30 \mu$ diameter laser spot, this represents a saturated mass detection of approximately 4 pg or 25 femto-moles per focal spot. For an NEOT of $0.3 \mathrm{~nm}$, the corresponding detection limit is $0.2 \mathrm{pg}$ or 1 femto-moles per focal spot.

The enhanced performance of an antinodal disk, that increases the field-dipole interaction by placing the molecular layer at a field maximum in the reflected standing wave, was verified and calibrated internally by the EO modulator. The effective optical thickness is increased by a factor of $2(n+1) \approx 4.8$ relative to the glass disk by this condition. Using this substrate, we detected a systematic shift of approximately $3.5 \mathrm{~nm}$ out of $10 \mathrm{~nm}$ in the biolayer thicknesses between FITC-conjugated and unconjugated avidin binding to a biotinylated surface. This relative measurement of the binding of two different types of avidin shows the sensitive capabilities of adaptive optical spinning-disk interferometry. The system cannot only detect biomolecules, but also can map out the distribution of different molecules across large areas in real-time with subnanometer accuracy. Therefore, the adaptive interferometric BioCD could be a powerful sensing technique for diagnostics as well as a new topography tool for surface science.

This work was supported by NSF grant ECS0200424, and by research sponsored by QuadraSpec, Inc. through the Purdue Research Foundation.

\section{References}

1. M. M. Varma, D. D. Nolte, H. D. Inerowicz, and F. E. Regnier, "Spinning-disk self-referencing inteferometry of antigenantibody recognition," Opt. Lett. 29, 950-952 (2004).

2. L. Peng, M. M. Varma, F. E. Regnier, and D. D. Nolte, "Adaptive optical biocompact disk for molecular recognition," Appl. Phys. Lett. 86, 183902 (2005).

3. A. Blouin and J.-P. Monchalin, "Detection of ultrasonic motion of a scattering surface by two-wave mixing in a photorefractive GaAs crystal,” Appl. Phys. Lett. 65, 932-934 (1994).

4. F. M. Davidson and L. Boutsikaris, "Homodyne detection using photorefractive materials as beamsplitters," Opt. Eng. 29(4), 369-377 (1990).

5. J. Khoury, V. Ryan, C. Woods, and M. Cronin-Golomb, "Photorefractive optical lock-in detector," Opt. Lett. 16, 1442-1444 (1991).

6. L. A. de Montmorillon, I. Biaggio, P. Delaye, J. C. Launay, and G. Roosen, "Eye-safe large field of view homodyne detection using a photorefractive CdTe:V crystal," Opt. Commun. 129, 293-300 (1996).

7. I. Rossomakhin and S. I. Stepanov, "Linear adaptive interferometers via diffusion recording in cubic photorefractive crystals," Opt. Commun. 86, 199-204 (1991).

8. R. K. Ing and J.-P. Monchalin, "Broadband optical detection of 
ultrasound by two-wave mixing in a photorefractive crystal," Appl. Phys. Lett. 59, 3233-3235 (1991).

9. I. Lahiri, L. J. Pyrak-Nolte, D. D. Nolte, M. R. Melloch, R. A. Kruger, G. D. Bacher, and M. B. Klein, "Laser-based ultrasound detection using photorefractive quantum wells," Appl. Phys. Lett. 73, 1041-1043 (1998).

10. B. F. Pouet, R. K. Ing, and S. Krishnaswamy, "Heterodyne interferometer with two-wave mixing in photorefractive crystals for ultrasound detection on rough surfaces," Appl. Phys. Lett. 69, 3782 (1996).

11. P. Yu, L. Peng, D. D. Nolte, and M. R. Melloch, "Ultrasound detection through turbid media," Opt. Lett. 28, 819-891 (2003).

12. S. Stepanov, V. Petrov, P. Rodriguez, and R. Lopez, "Directional detection of laser-generated ultrasound with an adaptive two-wave mixing photorefractive configuration," Opt. Commun. 187, 249-255 (2001).

13. P. Delaye, A. Blouin, D. Drolet, L.-A. Montmorillon, G. Roosen, and J.-P. Monchalin, "Detection of ultrasonic motion of a scattering surface by photorefractive InP:Fe under an applied dc field," J. Opt. Soc. Am. B 14, 1723-1734 (1997).

14. Q. Wang, R. M. Brubaker, D. D. Nolte, and M. R. Melloch, "Photorefractive quantum wells: transverse Franz-Keldysh geometry," J. Opt. Soc. Am. B 9, 1626-1641 (1992).

15. S. Balasubramanian, I. Lahiri, Y. Ding, M. R. Melloch, and D. D. Nolte, "Two-wave mixing dynamics and nonlinear hotelectron transport in transverse-geometry photorefractive quantum wells studied by moving gratings,” Appl. Phys. B 68, 863-869 (1999).

16. R. M. Brubaker, Q. N. Wang, and D. D. Nolte, "Nonlocal photorefractive screening from hot electron velocity saturation on semiconductors," Phys. Rev. Lett. 77, 4249-4252 (1996).

17. D. D. Nolte, T. Cubel, L. J. Pyrak-Nolte, and M. R. Melloch, "Adaptive beam combining and interferometry using photorefractive quantum wells," J. Opt. Soc. Am. B 18, 195-205 (2001).

18. B. J. Berne and R. Pecora, Dynamic Light Scattering: With Applications to Chemistry, Biology, and Physics (Dover, 2000).

19. M.-L. Theye, "Investigation of the optical properties of Au by means of thin semitransparent films," Phys. Rev. B 2(8), 3060 3078 (1970).

20. J. Duchet, J. F. Gérard, J. P. Chapel, and B. Chabert, "Grafting of alkylchlorosilanes ontosilica from solution for adhesion enhancement," J. Adhes. Sci. Technol. 14(5), 691-718 (2000).

21. W. Cho, "A new biocompatible coating for bioanalytical devices based on PSI (polysuccinimide)," Ph.D. dissertation (Purdue University, 2006).

22. E. Delamarche, A. Bernard, H. Schmid, B. Michel, and H. Biebuyck, "Patterned diversity of immunoglobulins to surfaces using microfluidic networks," Science 276, 779-781 (1997).

23. A. Bernard, J. P. Renault, B. Michel, H. R. Bosshard, and E. Delamarche, "Microcontact printing of proteins," Adv. Mater. 12, 1067-1070 (2000). 